\title{
First report of Periconia macrospinosa causing leaf necrosis of pointed gourd in India
}

\author{
Tanushree Sarkar $^{1} \cdot$ Prosenjit Chakraborty $^{1} \cdot$ Arup Karmakar $^{1} \cdot$ Aniruddha Saha $^{1} \cdot$ Dipanwita Saha $^{2}$ (D
}

Received: 31 August 2018 / Accepted: 3 June 2019 / Published online: 27 June 2019

(C) Società Italiana di Patologia Vegetale (S.I.Pa.V.) 2019

Keywords Pointed gourd $\cdot$ Necrosis $\cdot$ Periconia $\cdot$ Endophyte $\cdot$ Pathogenicity

Trichosanthes dioica, commonly known as pointed gourd, is one of the important vegetable crops of India. During the summer of 2017 cultivated pointed gourd plants with necrotic symptoms on leaves were found in sub-Himalayan region of West Bengal, India. Disease incidence was 20-25\%. Initially, yellow spots $(6-10 \mathrm{~mm})$ were observed on young leaves which gradually expanded and turned into grey rings with scattered tiny black dots. Subsequently, complete lamina became necrotic leading to defoliation. Isolation from diseased leaf samples yielded grey to black mycelia mat on PDA plates. Conidia were $18-35 \mu \mathrm{m}$ in diameter, dark brown to black, globose, borne singly or in short chains on simple or branched conidiophores and coarsely echinulate, spines, $2-7 \mu \mathrm{m}$ in length, adhered close to one another. Based on conidial morphology the isolate was identified as Periconia macrospinosa Lefebvre \& A.G. Johnson (Ellis 1968). The ITS and D1/D2 region of 28S rRNA large subunit (LSU) of representative isolate (PGISH2) were amplified using the primers ITS1/ITS4 and NL1/NL4 respectively and sequenced (GenBank accession No. ITS: MF447844 and 28S LSU: MF447843). These sequences showed 99\% sequence identity with ITS and 28S LSU region of P. macrospinosa strain CBS 135663 (ITS: KP183999 and 28S LSU: KP184038). Pathogenicity of the isolate was tested by spraying conidial suspension $\left(10^{6}\right.$ conidia/ $\mathrm{ml}$ ) onto both surfaces of the leaves of ten healthy twoweek-old potted pointed gourd plants. Control plants were sprayed with sterile distilled water. Necrotic symptoms, similar to those observed in the field, appeared on inoculated leaves after five days. Control plants remained symptomless. The fungus was successfully re-isolated from symptomatic tissues, fulfilling Koch's postulates. Although P. macrospinosa has previously been reported as a beneficial endophyte but in some cases it was established as a pathogen (Van Dyk 2004). In the present work, we found it as a pathogen causing necrosis. To our knowledge, this is the first report of $P$. macrospinosa causing leaf necrosis of pointed gourd in India.

\section{References}

Ellis MB (1968) Periconia macrospinosa. CMI Descr Pathog Fungi Bact $168: 1-2$

Van Dyk K (2004) Fungi associated with root and crown rot of wheat and barley in Tanzania. Afr Plant Prot 10:117-124

Publisher's note Springer Nature remains neutral with regard to jurisdictional claims in published maps and institutional affiliations.

Dipanwita Saha

dsahanbu@yahoo.com

1 Department of Botany, University of North Bengal, Darjeeling, West Bengal, India

2 Department of Biotechnology, University of North Bengal, Darjeeling, West Bengal, India 